\title{
Participación de actores sociales en gestión de programas estratégicos de salud en la región Huancavelica Perú
}

Participation of social actors in the management of strategic health programs in the Huancavelica region of Peru.

\section{MÁXIMO ALFONSO TUNQUE LIZANA}

Contador Público, Administrador de Empresas, Master en Gobierno y Gerencia en Salud, Maestro en Ciencias Empresariales, Maestro en Ciencias de la Educación, Doctor en Administración, docente invitado, correo electrónico institucional: maximo.tunque@unh.edu.pe, maxtunque@gmail.com, Universidad Nacional de Huancavelica, ORCID ID: https://orcid.org/0000-0003-3343-1715, Google académico https://scholar.google.es/citations?user=w4TPziMAAAAJ\&hl=es

\section{BENJAMÍN ROLDAN POLO ESCOBAR}

Ingeniero en Estadística e informática, Administrador, Magister en Dirección Estratégica en Tecnología de la Información, Magister en Gestión de los Servicios de Salud, Doctor en Administración de la Educación, Doctor en Gestión Pública y Gobernabilidad, Universidad Cesar Vallejo, Lima, Perú, benjamin.polo@untrm.edu.pe, brpoloescobar@gmail.com, ORCID: 0000-0001-5056-9957,

https://scholar.google.com/citations?view_op=list_works\&hl=es\&user=vNVEuPoAAAAJ

\section{HAYDEE EMILIA COLLANTES JIMÉNEZ}

Contador Público, Maestra en Gestión Pública, Jefa de Órgano de Control Institucional, Unidad de Gestión Educativa Local 12, hcollantes@ contraloria.gob.pe; urpiemilia@gmail.com, ORCID (0000-0002-6096-7083), https://scholar.google.com/citations?view_op=new_articles\&hl=es\&imq=Haydee+Emilia+ +Collantes+Jimenez+ORCID+(0000-0002-6096-7083)\#)

\section{HERBERT CHRISTIAN CORDOVA SOLIS}

Contador Público, Magister en Gestión Pública, Especialista Administrativo en el Ministerio Público, docente contratado, correo electrónico institucional: hccordovas@ucvvirtual.edu.pe, chris.cordovasolis@gmail.com, Universidad César Vallejo, ORCID ID: https://orcid.org/0000-0001-5764-8164, Google académico https://scholar.google.com/citations?hl=es\&user=8cT0WDYAAAAJ

\section{Revista Iberoamericana de la Educación \\ Vol - Especial 12021 \\ e-ISSN: 2737-632x}

\begin{abstract}
The purpose of this article was to analyze and explain the influence of the participation of social actors in the management of strategic health programs in the Huancavelica region of Peru, initially based on a background in the international and national context; subsequently, the conceptualization
\end{abstract}


of participation and management in health is made. The research methodology had a quantitative approach, a non-experimental design of descriptive explanatory cross-sectional level was used, the sample was made up of twelve social organizations and 368 collaborators who perform health functions to whom three questionnaires with 78 items were applied; for data analysis, the SPSS 25 program was used with a significance of 0.05 and for the statistical test, Simple Linear Regression was used with the Fisher's Pvalue approach. The results found were that there is a significant influence of the participation of social actors in the management of strategic health programs. In conclusion, the greater the participation of actors involved in health activities, the better the management of strategic programs, evidencing progress of tracer indicators.

Key words: Participation, social actors, public management, strategic programs.

Resumen: Los gobiernos regionales vienen implementando las políticas nacionales del Gobierno Peruano, en materia de la salud pública, el presente artículo tuvo como propósito analizar y explicar la influencia de la participación de actores sociales en la gestión de los programas estratégicos de salud en la región Huancavelica Perú, inicialmente se partió de unos antecedentes en el contexto internacional y nacional; posteriormente se realiza la conceptualización de la participación y la gestión en salud. La metodología de la investigación tuvo un enfoque cuantitativo, se utilizó un diseño no experimental de nivel descriptivo explicativo de corte transversal, la muestra fue realizada a doce organizaciones sociales y 368 colaboradores que cumplen funciones de salud a quienes se les aplicó tres cuestionarios con 78 ítems; para el análisis de datos se utilizó el programa SPSS 25 con una significancia de 0,05 y para la prueba estadística se utilizó la Regresión Lineal 
Simple con el enfoque $\mathrm{P}$ valor de Fisher. Los resultados encontrados fueron que existe una influencia significativa de la participación de actores sociales en la gestión de programas estratégicos de salud. Como conclusión a mayor participación de actores involucrados en el quehacer de salud mejora la gestión de programas estratégicos evidenciándose progreso de indicadores trazadores.

Palabras clave: Participación, actores sociales, gestión pública, programas estratégicos

\section{INTRODUCTION}

El Congreso de la República del Perú aprobó el Decreto Supremo N 0042013-PCM sobre la Política Nacional de Modernización de la Gestión Pública en Perú (El Peruano, 2013), marco normativo orientador de la gestión pública moderna que cuenta con cinco pilares centrales: políticas públicas y planes estratégicos, presupuesto para resultados, gestión por procesos y organización institucional, servicio civil meritocrático con la creación de SERVIR y un sistema de información seguimiento y monitoreo, cuyos ejes transversales como gobierno abierto, gobierno electrónico y articulación interinstitucional como fundamento para una gestión moderna orientada a resultados hacia el año 2021 .

El artículo plantea como problema fundamental ¿Por qué la participación de actores sociales influye en la gestión en salud a través de los programas estratégicos? en el logro de resultados tangibles hacia los usuarios externos y la ciudadanía. Actualmente el Estado Peruano viene implementando las intervenciones estratégicas en salud dirigidos a mejorar los procesos de intervención como el articulado nutricional con el objetivo de disminuir la desnutrición crónica infantil en menores de 5 años (Ministerio de Salud Perú, 2016a), salud materno neonatal para fortalecer los servicios de salud 
ofreciendo atención al niño y a la madre (Ministerio de Salud Perú, 2016b), control y prevención de la salud mental entre otros.

Es imperativo abordar la participación conjunta de actores sociales que intervienen en las estrategias para mejorar la salud como política de Estado y proponer alternativas de solución para la gestión de la salud, la importancia radica en la generación de conocimiento teórico considerando que el trabajo conjunto implicará una mejor atención en los servicios de salud, generando valor público al usuario. El propósito del estudio fue realizar el análisis y explicar sobre la influencia de la participación de los actores en las estrategias que viene implementado el Gobierno Peruano, cuáles son sus resultados de gestión y avances.

La presente investigación utilizó como soporte de la realidad dos variables: primero la participación de actores sociales para establecer la incidencia en la gestión de programas estratégicos de salud; en relación a la primera variable se fundamenta en la teoría de la acción comunicativa de Jürgen Habermas (Habermas, 2011) dicho filósofo en su obra establece tres mundos: el primer mundo objetivo el conglomerado de organizaciones para comunicarse, segundo el universo social en donde los actores interactúan a través de relaciones interpersonales, el tercero el mundo subjetivo donde se analiza al actor social como concertador e inteligente, a través de la interacción de miembros de un ente social los agentes se comunican a través del lenguaje para establecer objetivos comunes, coordinar acuerdos de apoyo mutuo para lograr metas, en ese orden de ideas los problemas más relevantes de salud como la desnutrición crónica infantil y la mortalidad materna neonatal por tratarse de cuestiones multicausales, serían necesariamente abordados por un conjunto de intervenciones que involucran diferentes instituciones llamados actores sociales para enfrentar los problema sociales. 
En este contexto (Lara, 2021) identificó dos maneras de participación: la institucionalizada y la comunitaria y "reconoce que la participación en salud tubo objetivos claros en el desarrollo de propuestas de políticas públicas y como parte de diferentes actores sociales han sido transformadores en las condiciones de vida de la comunidad" la misma autora complementa " la participación ciudadana vista desde la determinación social es entendida como la practica social enmarcada en las relaciones de poder", es necesaria el concurso de la ciudadanía en la gestión colaborativa para garantizar la mejora continua. Tal como afirma (Heredia \& Fonseca, 2020) se torna imprescindible el concurso de quien "debe liderar y construir en equipo que va a soportar las medidas de acción que deben ser tomadas en la organización para alcanzar las metas propuestas en el marco de una administración colaborativa" se debe interactuar en entornos colaborativos como estrategias; son desafíos que enfrentan las actuales organizaciones en el que una administración participativa con cuyos integrantes que deben participar para tomar decisiones constituyéndose como un actor importante del cambio con metas claras. Para (Idrogo et al., 2020) "en América Latina se viene promoviendo la participación involucrando a la población mediante los presupuestos participativos, en la planificación de los recursos del Estado con el propósito de mejorar los proyectos de interés común" el Estado promueve la participación considerando instrumento de política y gestión dentro del plan de desarrollo concertado. También nos dice (Dueñas \& García, 2012) que " la participación es asumida como una forma de comprender las relaciones sociales para afrontar la realidad y dar solución a los problemas" en el contexto de los programas estratégicos los actores involucrados se reúnen periódicamente para tratar asuntos de la intervención en salud de manera conjunta. Al respecto de la participación en gestión Willian Ouchi en su modelo de trabajo cooperativo llamada Teoría $\mathrm{Z}$ menciona que la 
organización impulsa las relaciones cooperativas y el trabajo colectivo consensuado, interacción en grupo para una adecuada toma de decisiones.

Otro componente de participación es el gobierno abierto como forma de intervención, la ciudadana colabora y coopera produciendo sinergias organizacionales con sus propuestas en el planteamiento de normas, controla y realiza seguimiento de los quehaceres de la salud por parte del Estado que promueve el dialogo sobre acuerdos y compromisos en la gestión, conoce sus derechos, acceso a la información de carácter público al mismo tiempo que el usuario externo se informa sobre los acontecimientos necesidades en la prestación de servicios públicos como forma de cumplimento de las políticas sociales". De la misma manera (Núñez et al., 2021) reconocen “dos modalidades de participación: la directa y la indirecta. La modalidad directa es la más fidedigna expresión de la participación en función del cumplimiento de los principios cooperativos. La modalidad de participación que incide en la gestión cooperativa".

En relación a la segunda variable del estudio gestión de programas estratégicos de salud como estrategias nacionales implementados en el Perú por el Ministerio de Salud desde el año 2008, para dar solución a problemas en la salud pública y que necesariamente debe ejecutarse con la intervención articulada de actores en los diferentes niveles de la administración, con el fin de lograr resultados que se esperan obtener por metas definidas que necesariamente implican un cambio del estilo de vida como impacto y finalmente crean valor público al usuario (Alvarez \& Gálvez, 2021). Al respecto (Melgar Carrasco, 2019) considera que "los servidores deben orientarse, en el marco de las políticas públicas de Estado y las responsabilidades de cada sector, en atender las necesidades de los usuarios; organizando los procesos de transformación de los insumos en servicios de calidad en todo el proceso de la cadena de valor" acota el mismo autor "que 
se traduzcan en una mayor satisfacción de los usuarios y que garanticen sus derechos a un servicio de salud de calidad en un tiempo oportuno y al menor costo posible", pero para lograr todo lo anterior consideramos que la gestión debe fortalecer estructuras y herramientas contenidas en los componentes de gestión para resultados como: continuar con la implementación de una planeamiento estratégico en un horizonte de largo plazo actualizando los planes estratégicos cada cuatro años y los planes operativos de manera semestral, fortalecer el proceso de control gerencial a través del monitoreo y supervisión de actividades de salud así como la evaluación trimestral de avances y resultados para ajustar las desviaciones juntamente con los actores involucrados, implementar la mejora continua de la gestión por procesos haciendo cambios en la estructura plasmando proyectos de mejora para obtener ventaja competitiva. Otro componente estratégico es el uso de los sistemas de información gerencial para toma de decisiones en salud con eficiencia y eficacia, pero con un sistema único, no como el actual sistema absolutamente fragmentados que no permiten obtener información oportuna y confiable, otro aspecto relevante es la medición de los resultados de salud a través de evaluación de actividades.

La gestión del capital humano competente ayudará en el logro de objetivos institucionales, se ha vuelto un reto en las organizaciones de hoy conforme detalla (López, 2018) las competencias que debe contar los colaboradores son herramientas útiles para lograr el objetivo, empero para llevar a cabo la práctica es fundamental contar con la alta dirección para guiar a los equipos de trabajo en la consecución de metas de manera conjunta. Es imperioso de acuerdo a (Robbins \& Judge, 2017) fortalecer las competencias del capital humano en salud a través de acciones de capacitación con el uso de entornos virtuales aprovechando la cooperación externa y la universidad, mantener siempre motivado a los colaboradores para mejorar el compromiso 
organizacional, el que conlleva a una mayor satisfacción laboral, participación e involucramiento en las labores. Para que la gestión en salud logre mejorar sus indicadores es condición que el reclutamiento del capital humano en salud se realice a través de una meritocracia para captar a los mejores profesionales con un adecuado diseño de puestos y evaluación del desempeño laboral con incentivos y recompensas por el compromiso mostrado en la mejora de procesos (Jara, 2018).

Se examinó el estado de arte previo en el contexto internacional como: (Roa, 2013) considera existe marcado interés conforme a las políticas públicas para incorporar a los actores sociales en la intervención social "su opinión, inquietudes, intereses. muchas veces, esta intención aparece bajo la categoría de participación, la que intenta ser instalada como modo de hacer en procesos desplegados por el estado, por privados y su RSE, por gobiernos locales y ONGs”. (Valenzuela, 2019) estableció en México que si a la ciudadanía se les convoca desde el gobierno "bajo parámetros institucionales, si la invitación a participar viene desde la sociedad civil, entonces se concibe desde otro parámetro, más ciudadano y enfocado a sus problemáticas” y respecto a las modalidades de participación de ciudadanos son extensas y se pueden forjar dentro y fuera del gobierno, al mismo tiempo que permite generar mayor confianza y colaboración entre organizaciones. Así mismo (Paricio-Esteban et al., 2020) sobre transparencia y participación ciudadana del Gobierno de España considera el reconocimiento de "5 modelos sobresalientes de webs y portales de transparencia: web independiente, apartado en web, sede electrónica, plantilla de la Diputación provincial y plantillas". Mientras que (Aracena Genao et al., 2020) con respecto a la implementación de la Nueva Gestión Pública en hospitales de México se pudo evidenciar "el alto rendimiento, constante durante todo el período analizado, los hospitales de la Secretaría de Salud alcanzaron, y conservaron, niveles 
óptimos de efectividad y eficiencia". Señala (Montes de Oca, 2019) en el mismo país respecto al mecanismo de participación como espacio de transparencia control social en gestión pública se gobierna a través de consejos consultivos en la toma decisional como descentralización de poder. Acotan (Folleco \& Legarda, 2020) complementando la diferencia entre gobernanza y NGP puntualizan en que la primera "privilegia la participación del mercado en la prestación de servicios públicos; en tanto que, la segunda apunta a la intervención de todos los actores sociales en los procesos", (Rivera, 2021) da a conocer respecto a factores predominantes en la gestión pública se da en base a participación ciudadana lo cual es importante establecer una valoración de los cuatro procesos: planificación, organización, dirección, control y los "factores de gobiernos participativos con la colectividad como son: consejos de planificación, presupuestos participativos, consejos barriales o parroquiales, mesas de diálogo y finalmente la rendición de cuentas". Por su parte (Castro et al., 2020) considera importante que con opinión de los usuarios a través de la participación ciudadana ayudaran a mejorar los procesos al permitir conocer sus demandas y necesidades, instrumento que mejora la gobernanza local y toma de decisiones". Así mismo (Cedillo \& Rivadeneira, 2020) afirma con respecto a la ciudadanía en educación "participación que debe transitar por la preparación de la sociedad para el ejercicio democrático de este derecho, de manera activa". También (Medranda et al., 2019) detallan que el gestor público está obligado a mostrar resultados tangibles activando la participación ciudadana y que la transparencia es primordial para promover la participación de actores locales, sin embargo contrapone que en Ecuador se constata respecto a no existencia de "mecanismos suficientes para el despliegue de una participación ciudadana democrática y aceptable que propicie ambientes de crecimiento y desarrollo social y económico, sostenible 
y sustentable en el tiempo", se hace necesario fortalecer competencias a la sociedad a fin de que ponga en práctica el uso y goce de sus derechos y facultades para colaborar en la gestión pública. Mientras que (Contreras, 2018) encontró que existe un reducido grupo de indicadores orientados al éxito mediante la consecución de resultados tangibles en usuarios externos de los programas implementados, con el consiguiente privilegio mayoritario hacia los indicadores de gestión dependientes directamente del servicio prestado. Por lo que, (Milanesi, 2018) considera que la "gestión por Resultados (GpR) es una idea dominante de reforma del sector público. Sin embargo, los factores que condicionan su desarrollo no han sido suficientemente debatidos en América".

Mientras que los trabajos previos nacionales: Con respecto a la participación (Flores Mamani et al., 2020) manifiestan una buena precepción sobre la participación de las organizaciones de base para mejorar la gestión de las políticas públicas caso Salcedo-Puno. También (Príncipe, 2021) en su estudio sobre participación en gobiernos locales del Perú considera que a mayor participación conjunta ciudadana "mejor se desarrollan los procesos de presupuesto participativo. Se afirma que existe una relación positiva significativa entre la participación ciudadana y el presupuesto participativo". En relación a la gestión pública: en su estudio (Zegarra et al., 2021) respecto al valor público en la gestión reflexiona que los gestores deben prestar los "servicios públicos acorde a sus posibilidades, con beneficios y niveles de agrado para el poblador, es preciso conocer las insuficiencias de los protagonistas favorecidos para conseguir valorar los horizontes de agrado con la eficacia de los productos y servicios”. Por su parte, (Villegas-flores, 2021) afirma en su estudio que "se ha determinado que existe una relación significativa entre el Presupuesto por Resultados y la gestión administrativa en la Unidad Ejecutora $N^{\circ} 001230$ " realizado en la Universidad Nacional de 
Moquegua, a mayor presupuesto mejora la gestión. Así mismo, (Melgar Carrasco, 2019) considera que "la política pública, el planeamiento y el presupuesto por resultado vienen influyendo significativamente en la reforma de salud" acotando que los sus resultados aún no se visibilizan en el corto plazo, y se espera evidenciarán el logro de objetivos y mejoramiento de procesos en el mediano y largo plazo. También (Guerra \& Palomino, 2020) en la UGEL San Martín Perú se encontraron claras evidencias de "procesos deficientes en la ejecución de gasto, estos no solo en la percepción de los diversos especialistas, sino también en los que conforman dichas unidades como son los directores, entonces el desarrollo de un modelo de gestión contribuye significativamente para la mejora de acciones, procesos $\mathrm{o}$ elementos de cambio”. Según (Huanca, 2019) respecto a la aplicación del presupuesto por resultados en gobiernos locales del Estado Peruano se logró mejorar la calidad del gasto público porque fueron mayormente son destinados a favorecer los niveles de la calidad de vida de la población usuaria. Por su parte, (Chura, 2021) encontró que "la gestión por resultados de la ejecución presupuestal influye positivamente en la Gestión Financiera de logro de metas físicas programadas y objetivos institucionales de la UGEL de Puno". Mientras que, (Palomino, 2021) sostiene que de acuerdo al análisis del impacto de gestión por resultados respecto a la satisfacción de la calidad de atención de los procesos contrariamente "los usuarios no están satisfechos en la calidad del servicio, por incumplimiento de plazos, indiferencia y mal desempeño de algunos funcionarios". (Barros Bastidas \& Turpo Gebera, 2017)Mientras que, (Vargas Merino \& Zavaleta Chávez, 2020) consideran que se ha logrado un avance significativo en la implementación de los principales instrumentos de la reforma en los gobiernos locales del Perú, pero la repercusión para orientar el proceso presupuestario hacia un enfoque por resultados todavía es limitada, complementan "los programas presupuestales, 
y el seguimiento de su desempeño, presentan deficiencias en su implementación y no cuentan con mecanismos efectivos". Finalmente, (Beltrán et al., 2020) encontró que el "impacto de la ejecución de gasto en la desnutrición crónica determino que los distritos de Yúngar y Anta, considerados como orientados a la gestión por resultados, lograron un mayor nivel de variación porcentual de la desnutrición crónica en $24.14 \%$, a diferencia en promedio de $10.55 \%$ respecto a los distritos no calificado como orientados a la gestión por resultados".

\section{Materiales y métodos}

En la investigación se utilizó el método científico, con un enfoque cuantitativo de tipo teórico básico, realizado con el nivel explicativo descriptivo, para responder a la pregunta de investigación se planteó un diseño de investigación no experimental de corte transversal fundamentado en (Hernández-Sampieri \& Mendoza, 2018), complementado con métodos de análisis, deducción, abstracción para modelar los constructos teóricos y medición para comparar las propiedades de las dimensiones estudiadas.

Se tomó en consideración una población finita representada por 8743 actores funcionarios y colaboradores cuyos criterios de inclusión fueron: efectuar actividades de salud y el de exclusión no desarrollar actividades de salud conformada por doce unidades de análisis de instituciones públicas que implementan acciones de salud de manera directa e indirecta en el ámbito de intervención región Huancavelica: El Ministerio de Salud, Gobierno Regional, Dirección Regional de Salud, redes de salud, Hospital Regional Zacarías Correa Valdivia, hospitales provinciales de Pampas, Acobamba y Lircay, instituciones prestadoras de salud públicas, Dirección Regional de Educación, Unidad Desconcentrada Regional Seguro Integral de Salud, gobiernos locales, Programa de Apoyo Directo a los más Pobres y el Programa Nacional de Alimentación Escolar. 
Se analizó una muestra estadística probabilista aleatoria de tipo estratificado de doce instituciones públicas constituida por 368 trabajadores del sector público de la Región Huancavelica a 3680 m.s.n.m. personal de salud de redes y hospitales, autoridades decisores del gobierno regional, local y programas sociales que intervienen en la región, gerentes, sub gerentes, directores, alcaldes y coordinadores de gestión en salud.

Para la recolección de datos de opinión de la población objetivo se utilizó la técnica de la entrevista con su instrumento de recolección de información la encuesta a través de tres cuestionarios nuevos de elaboración propia con cinco categorías de la escala de Likert: dos cuestionarios de 24 reactivos cada uno para la primera variable participación de actores sociales y uno de 30 ítems para la segunda variable gestión en salud todos con cinco opciones ("Nunca", “Casi nunca”, “A veces”, “Casi siempre” y "Siempre”). Se realizó la validez de contenido del instrumento de recolección para determinar la objetividad, coherencia y veracidad con juicio de cinco expertos investigadores reconocidos con grado de doctor de la Universidad Nacional del Centro del Perú, Universidad Peruana Los Andes y Universidad Nacional de Huancavelica; así mismo, la confiabilidad de los instrumentos se determinó con el estadístico Alfa de Cronbach con prueba piloto al $20 \%$ de la muestra para establecer la consistencia de las respuestas y no presenten variaciones significativas; en los tres cuestionarios se encontraron los coeficientes de 0,94, 0.94 y 0,95 considerados confiabilidad muy buena. La encuesta fue aplicada por siete encuestadores capacitados en un período de cuatro meses debidamente identificado con credenciales credenciales. Se complementó con la técnica del análisis documental a través de matrices para resumir el marco conceptual y el estado del arte objeto del estudio.

Como procedimientos para el trabajo de campo se exploró la data y se clasificó los cuestionarios con contenido de variables cualitativas, se validó 
los instrumentos interna y externamente para garantizar la objetividad, luego se planificó para el recojo de datos, se coordinó con los responsables de las entidades sobre los permisos por escrito para las visitas, se realizó el análisis documental de la información en forma paralela, luego se procedió a aplicar los cuestionarios a cargo de encuestadores por cada provincia: Huancavelica, Acobamba, Angaraes, Castrovirreyna, Churcampa, Tayacaja y Huaytará; se tuvo dificultades con los gobiernos locales provinciales y programas sociales seleccionadas como muestra al no encontrárseles en el centro laboral, se tuvo que retornar en forma reiterada. El tiempo utilizado para la recolección de datos fue de cuatro meses entre enero a abril del año 2017.

Para procesar la información recogida en el trabajo de campo mediante los cuestionarios, una vez codificados, clasificados y organizados se recurrió a las herramientas estadísticas software SPSS versión 24 para el análisis cuantitativo, se empleó la estadística descriptiva para cada variable de estudio estableciendo las tablas de frecuencias, medidas de tendencia central, desviación estándar y varianza a fin de entender el comportamiento de datos y plasmar en tablas y gráficas. Para realizar las pruebas de la hipótesis planteada se utilizó las estadísticas inferenciales; previamente se realizó el análisis paramétrico a través de la prueba de normalidad KolmogorovSmirnov. Se utilizó la regresión lineal simple para determinar la dependencia de las variables, con un nivel de significancia del 5\%, calculado a través del estadístico de regresión lineal simple con el enfoque P valor de Fisher para la decisión con cinco pasos, estableciendo una significancia menor a 0,5 para aceptar la hipótesis de estudio, a un nivel de confianza de 95\%, asimismo se encontró una relación fuerte de variables coeficiente correlación positiva fuerte de 0,84 y $r$ cuadrado ajustado de 0,71 en ambos casos fuerte aceptable por tratarse de variables de estudio cualitativas el que permitió medir las implicancias de la participación de actores sociales en la gestión de salud. 


\section{Resultados}

Para determinar los resultados se aplicó en un primer momento la estadística descriptiva para las variables y sub variables de estudio, en un segundo momento la estadística inferencial para la prueba de hipótesis.

Se caracterizó a los actores sociales en la región Huancavelica Perú que conformaron la muestra estadística del estudio de 368 colaboradores de doce instituciones públicas detallados consiguiente:

Figura 1. Caracterización de actores sociales de la región Huancavelica Perú

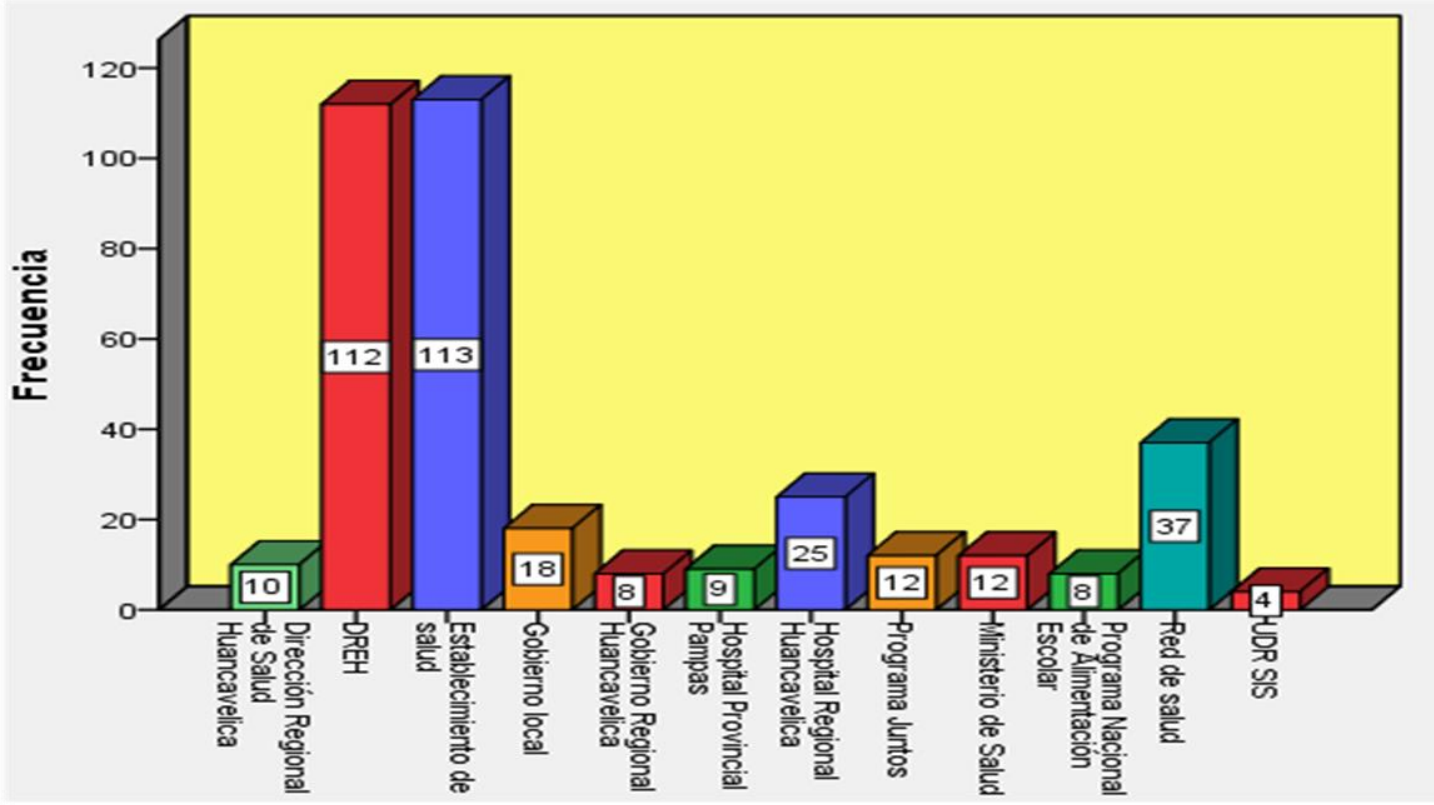

En la figura 1 se detalló a los actores participantes en el estudio de una población de 12 instituciones públicas los cuales representa a 8743 se estableció una muestra estadística de 368 colaboradores con un nivel de confianza del 95\%, de los seleccionados la muestra de 113 y 112 representan $30 \%$ del total muestreado establecimientos de salud y unidades de gestión locales de Educación, mientras que 37 trabajadores de las redes de salud 10\%, Hospital Regional Huancavelica 7\%, gobiernos locales 5\%, Dirección Regional de Salud 3\% y el resto de instituciones seleccionadas 2\% respectivamente. 
Figura 2. Resumen de frecuencias de la variable participación de actores sociales

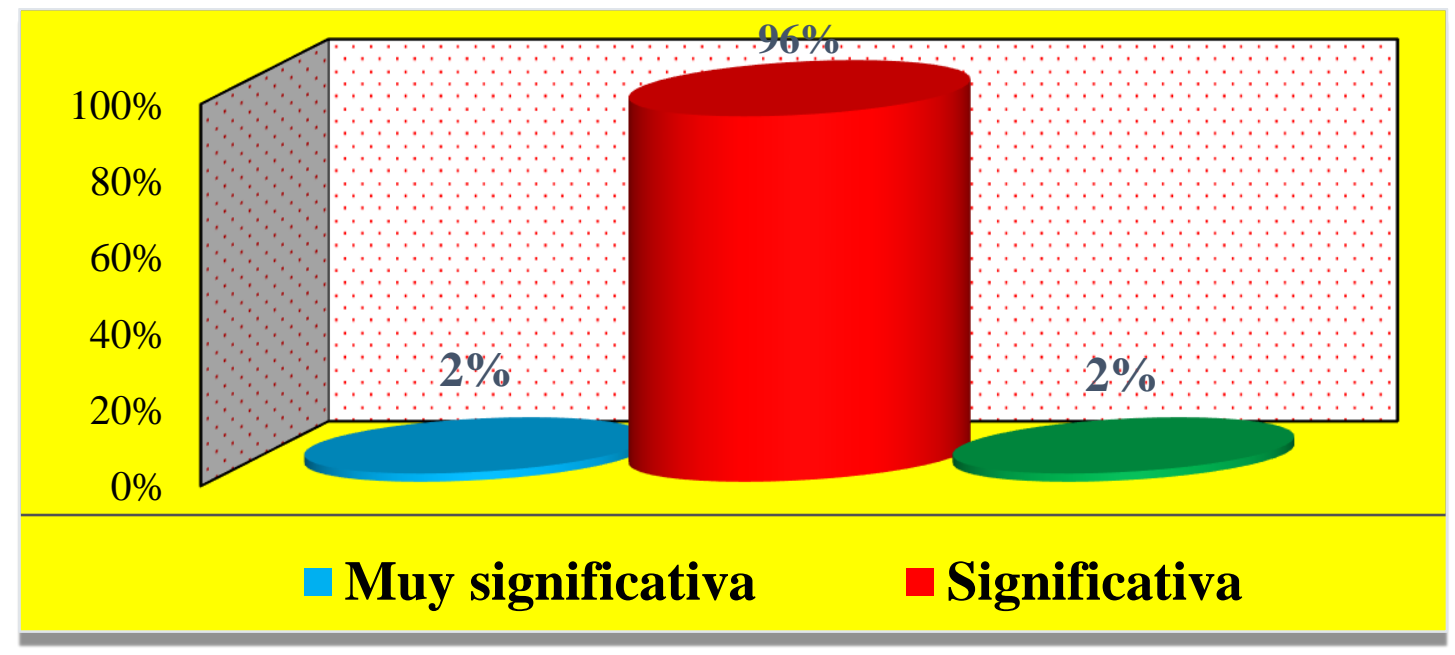

Realizado el análisis de la data aplicada con los cuestionarios con estadísticas descriptivas con las categorías y escalas: "siempre 5", “casi siempre 4", “a veces 3", “casi nunca 2" y "nunca 1" se muestran los resultados de la primera variable y se aprecia que la mayoría respondieron entre 3, 4 y 5 y que representan el $98 \%$, mientras que solo un $2 \%$ contestaron en el rango de categorías 1 y 2 respectivamente.

Figura 2. Resumen de frecuencias de la variable gestión de programas estratégicos de salud 


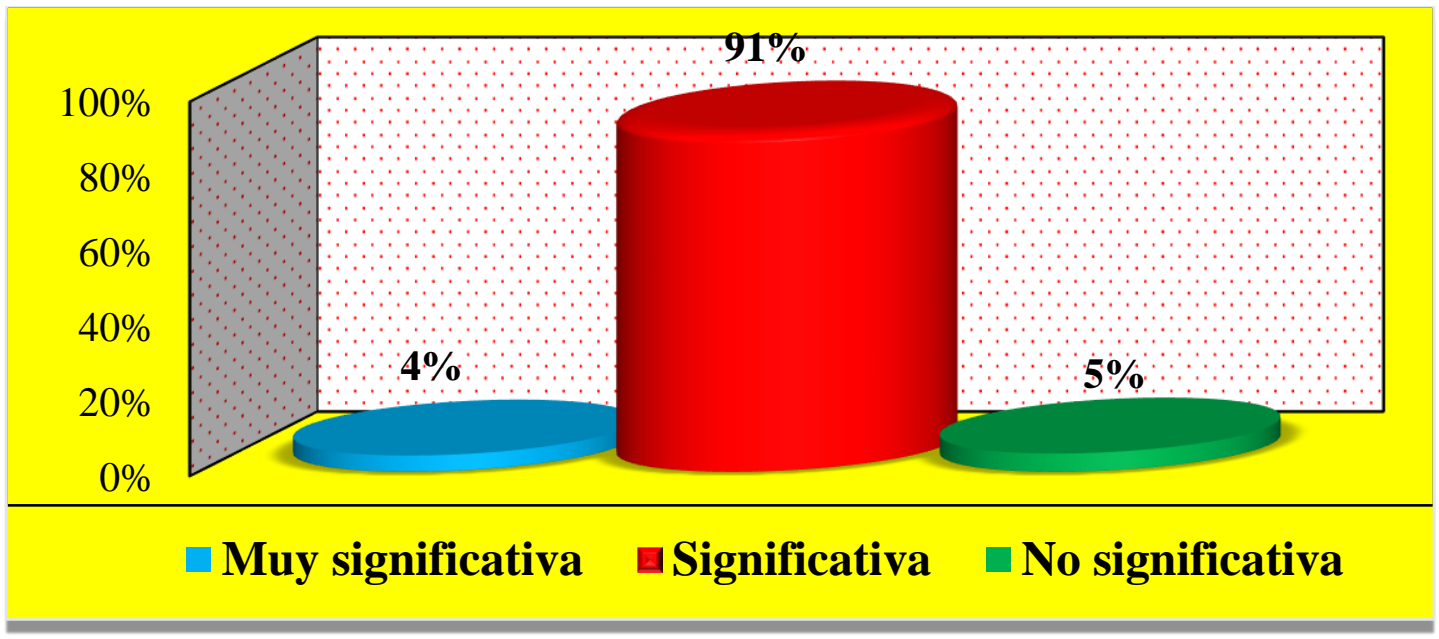

Realizado en procesamiento de la data aplicada con los los instrumentos con el uso de estadísticas descriptivas con las categorías y escalas: "siempre 5", “casi siempre 4", “a veces 3", “casi nunca 2" y "nunca 1" se muestran los resultados de la segunda variable y se aprecia que la mayoría respondieron entre 3, 4 y 5 y que representan el $95 \%$, mientras que solo un $4 \%$ contestaron en el rango de categorías 1 y 2 respectivamente.

Para la presentación de resultados inferenciales primeramente, se realizó la prueba de normalidad para determinar si los datos proceden de una distribución para ello se planteó la siguiente hipótesis nula: Ho: Los datos provienen de una población con distribución normal, se estableció con la prueba de Kolmogorov-Smirnov para una muestra cuyo resultados de ambas variables fueron significancia de 0,075 y 0,20 en ambos casos el $\mathrm{P}$ valor de Fischer resulta que $\alpha .=0,05$; por lo tanto, los datos provienen de una distribución normal. Consecuentemente procedemos a realizar las pruebas de hipótesis con el estadístico Regresión Lineal Simple.

\section{Hipótesis general de investigación}

Se ha tomado en consideración los cinco pasos establecidos por (Alvarado \& Agurto, 2013), el primero plantear la hipótesis nula Ho y la hipótesis alterna 
Ha, segundo seleccionar el nivel de significancia $\alpha$, tercero establecer el estadístico de prueba para determinar el P Valor: Se utilizó Anova y prueba F y como regla de decisión el $\mathrm{P}$ valor de Fisher, cuarto formular la regla de decisión y quinto tomar una decisión.

Ho: La participación de actores sociales no influye en la gestión de programas estratégicos de salud en la región Huancavelica.

Hi: La participación de actores sociales influye significativamente en la gestión de programas estratégicos de salud en la región Huancavelica.

Tabla 1

Prueba de regresión lineal de participación de actores sociales en la gestión de programas estratégicos de salud

\begin{tabular}{|c|c|c|c|c|c|c|c|c|}
\hline & $\mathrm{S}$ & & & & & & & \\
\hline & & $\mathrm{u}$ & & & $\mathrm{e}$ & & & \\
\hline & & $\mathrm{m}$ & & & d & & & \\
\hline & & $\mathrm{a}$ & & & $\mathrm{i}$ & & & \\
\hline & & $d$ & & & $a$ & & & \\
\hline & & e & & & $c$ & & & \\
\hline & & $\mathrm{c}$ & & & $\mathrm{u}$ & & & \\
\hline & & $\mathrm{u}$ & & & $a$ & & & \\
\hline & & $\mathrm{a}$ & & & d & & & \\
\hline & & d & & & $r$ & & & \\
\hline & & $\mathrm{r}$ & & & á & & & \\
\hline Mod & & $\mathrm{a}$ & & & $\mathrm{t}$ & & S & \\
\hline $\mathrm{e}$ & & d & & & $\mathrm{i}$ & & & i \\
\hline 1 & & O & $\mathrm{g}$ & & $\mathrm{c}$ & & & g \\
\hline O & & $\mathrm{S}$ & & 1 & $\mathrm{a}$ & $\mathrm{F}$ & & . \\
\hline
\end{tabular}




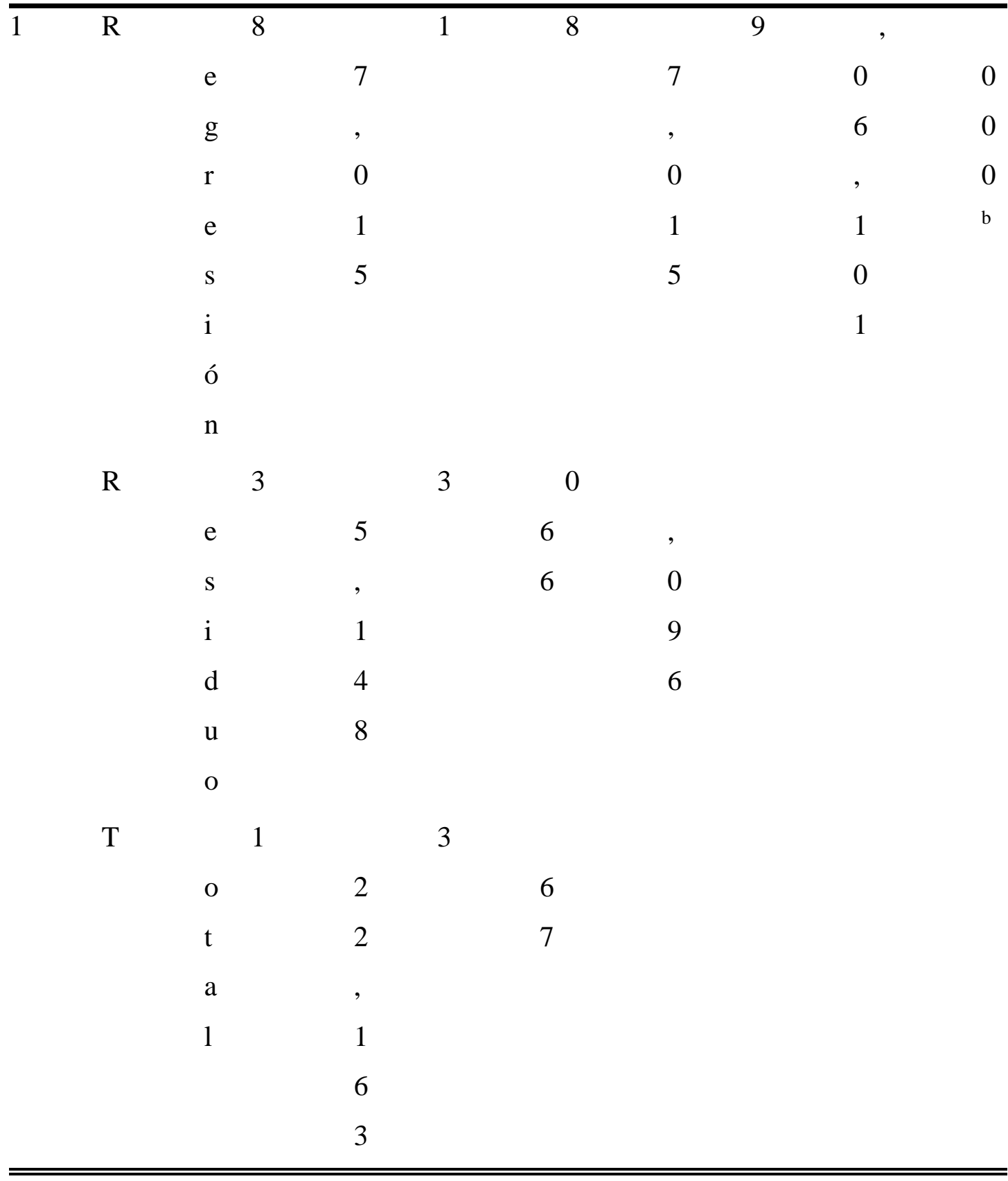

En la tabla 1, se muestra el modelo predictivo $\mathrm{Y}=\mathrm{f}(\mathrm{x})$ Regresión Lineal Simple, comprobamos el valor $\mathrm{P}=0,00$ menor a la significancia $=0,05$ por lo que, se rechaza la hipótesis nula y aceptamos la hipótesis de investigación. 
Se concluye que la variable participación de actores sociales tiene un poder explicativo significativo para explicar la segunda variable gestión de programas estratégicos de salud en la región de estudio Huancavelica Perú. Los predictores encontrados fueron el Coeficiente de Correlación $\mathrm{R}=0,84$ positiva fuerte, mientras que $r^{2}$ ajustado $=0,71$ también fuerte lo que permite afirmar influencia estadística y significancia global entre las variables de estudio, así mismo el 71\% de las variaciones en la gestión de los programas estratégicos es influenciada por la participación de actores sociales y el resto por variables o dimensiones desconocidas motivo de otras investigaciones subsiguientes.

Tabla 2

Prueba de regresión lineal de variables y dimensiones

\begin{tabular}{|c|c|c|c|c|c|c|c|c|c|c|}
\hline \multirow{16}{*}{ H } & & $\mathbf{P}$ & $\mathbf{P}$ & & & & $\mathbf{P}$ & & $\mathbf{P}$ & \\
\hline & $\mathbf{a}$ & & $a$ & $\mathbf{a}$ & $\mathbf{P a}$ & & & a & & \\
\hline & $\mathbf{r}$ & & $\mathbf{s}$ & $\mathbf{S}$ & $\mathrm{s}$ & $\mathbf{S}$ & & $\mathrm{s}$ & & \\
\hline & $\mathbf{i}$ & & C & $\mathbf{0}$ & & $\mathbf{0}$ & & 0 & & \\
\hline & $\mathbf{a}$ & & 1 & 2 & 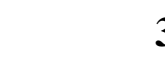 & 3 & & 4 & & \\
\hline & b & & & & & & & & & \\
\hline & 1 & & & & & & & & & \\
\hline & $\mathbf{e}$ & & & & & & & & & \\
\hline & $\mathbf{s}$ & & & & & & & & ) & \\
\hline & $\mathbf{y}$ & & & & & & & & & e \\
\hline & d & & & $P$ & & & & & & c \\
\hline & & $\mathbf{H}$ & & & $\mathbf{v}$ & & & & & i \\
\hline & $\mathbf{m}$ & & 0 & & $\mathbf{a}$ & & & & & $\mathbf{s}$ \\
\hline & e & & y & & l & & $p$ & & & i \\
\hline & $\mathbf{n}$ & & $\mathbf{I}$ & & $\mathbf{0}$ & $r$ & & $\mathrm{y}$ & & $\mathbf{0}$ \\
\hline & $\mathbf{S}$ & & a $\alpha$ & & $\mathbf{r}$ & & & $\alpha$ & & $\mathbf{n}$ \\
\hline
\end{tabular}




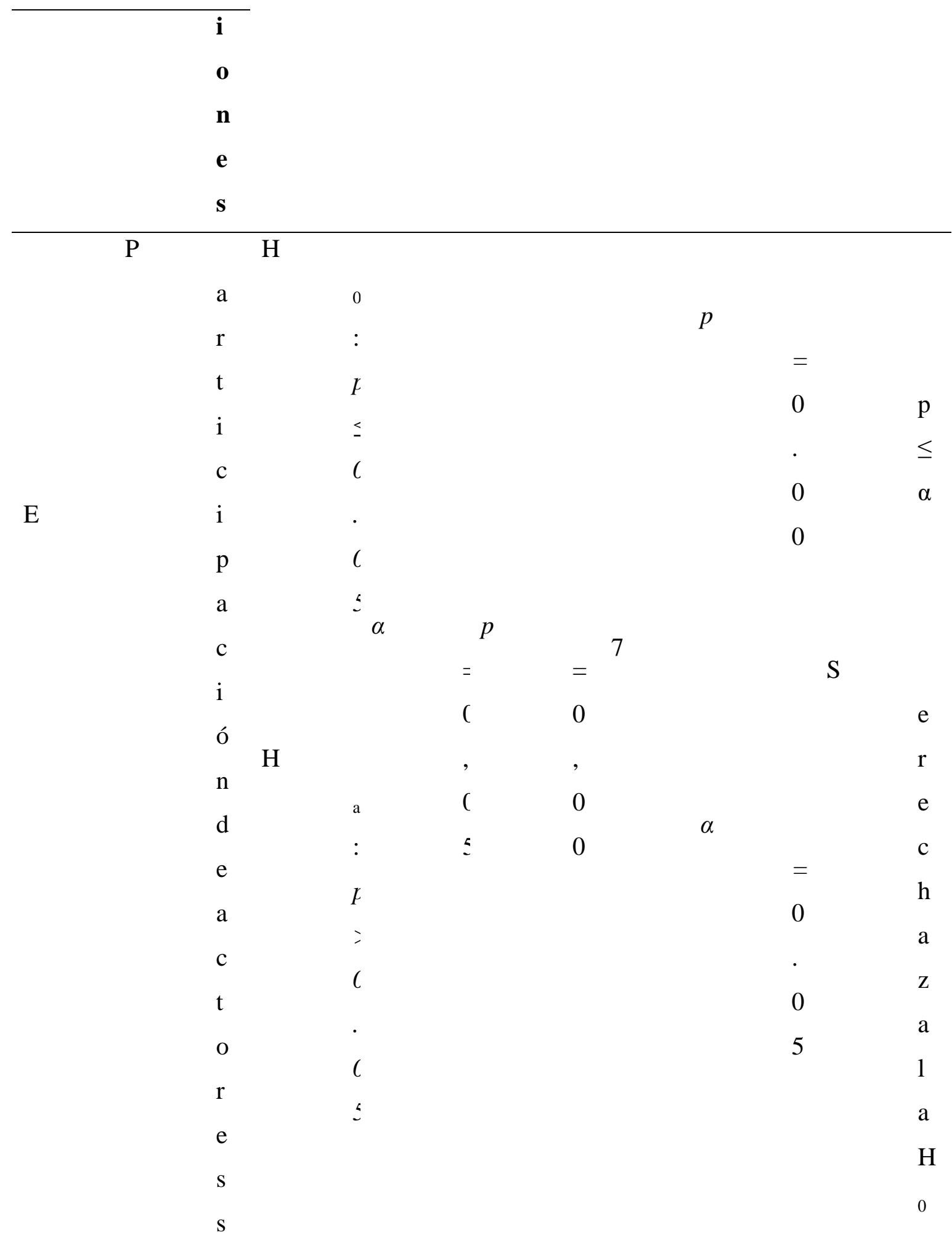


Formación docente revista iberoamericana de educación

O

c

i

a

1

e

S

$\mathrm{y}$

c

O

m

$\mathrm{p}$

O

n

e

n

t

e

S

d

e

g

e

S

$\mathrm{t}$

i

Ó

n 


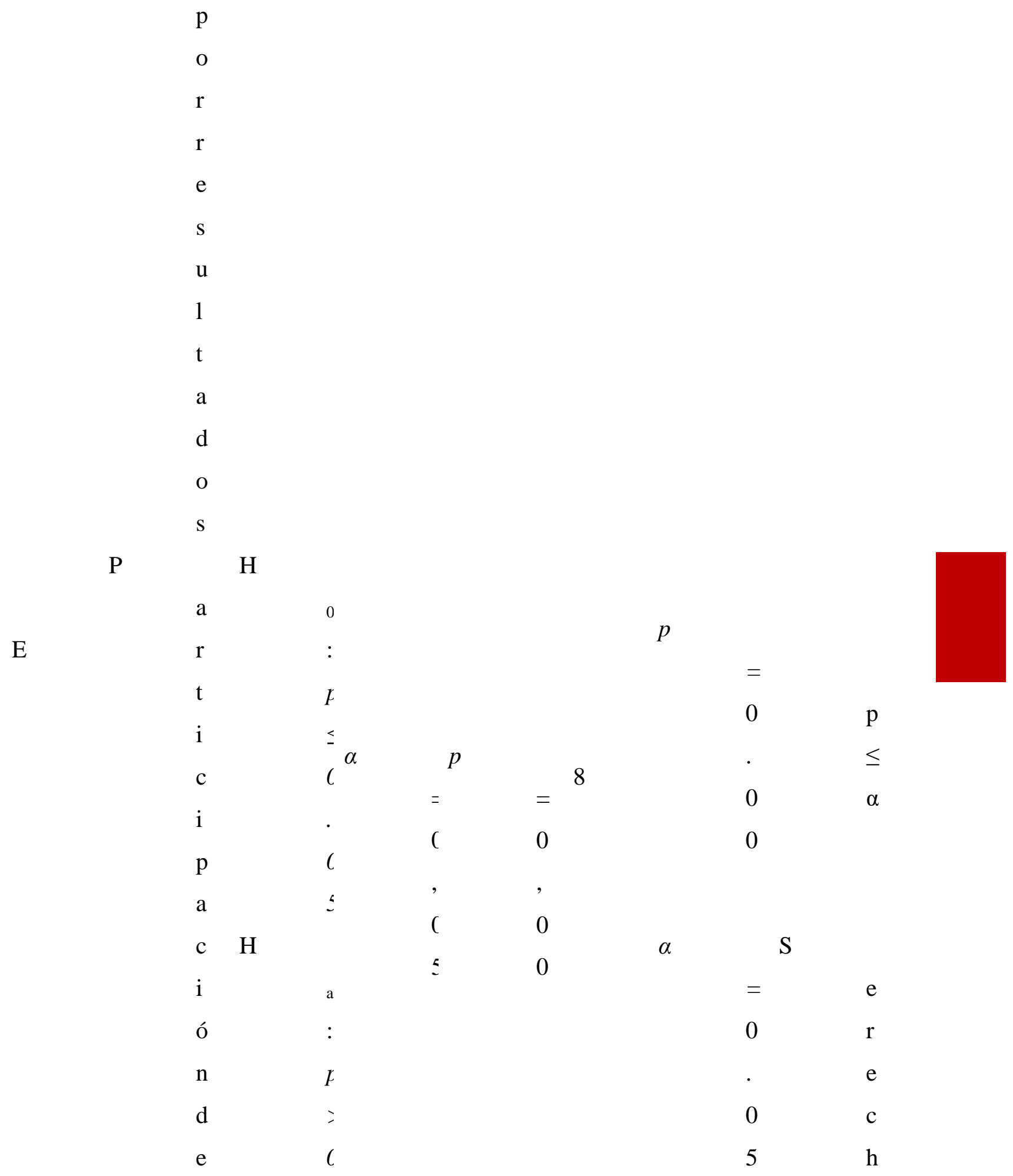


O 


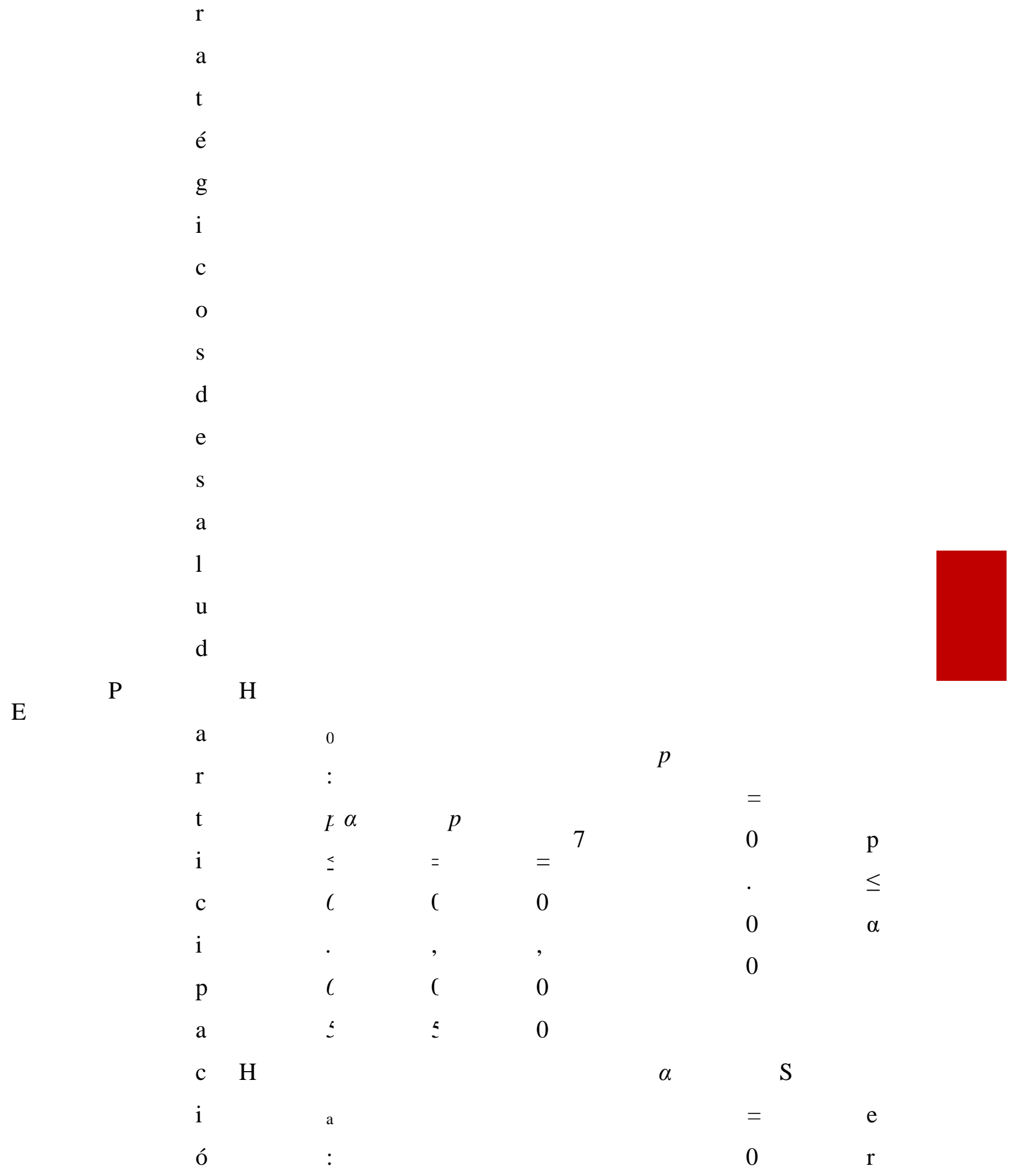


Formación docente

revista iberoamericana de educación
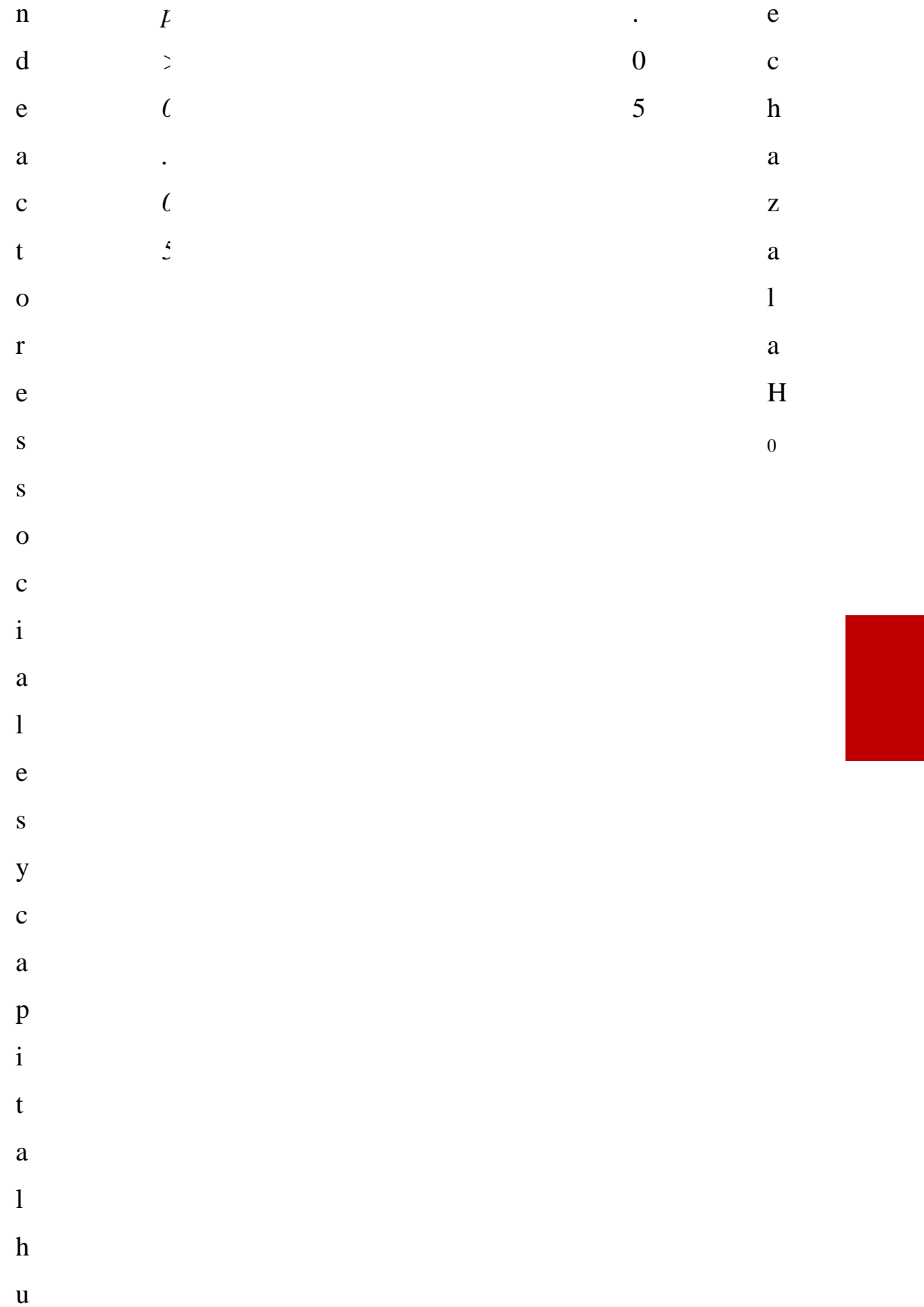


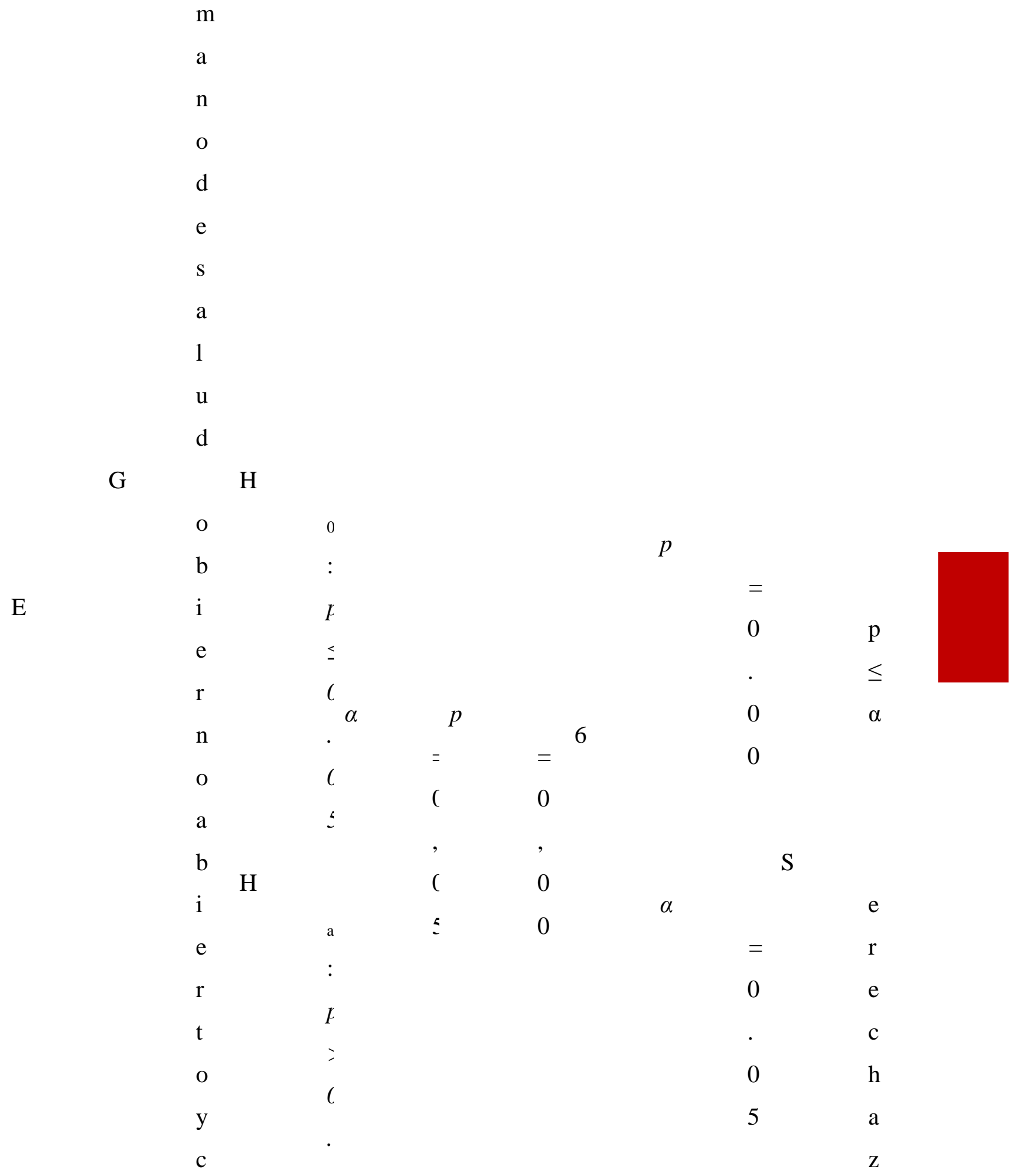




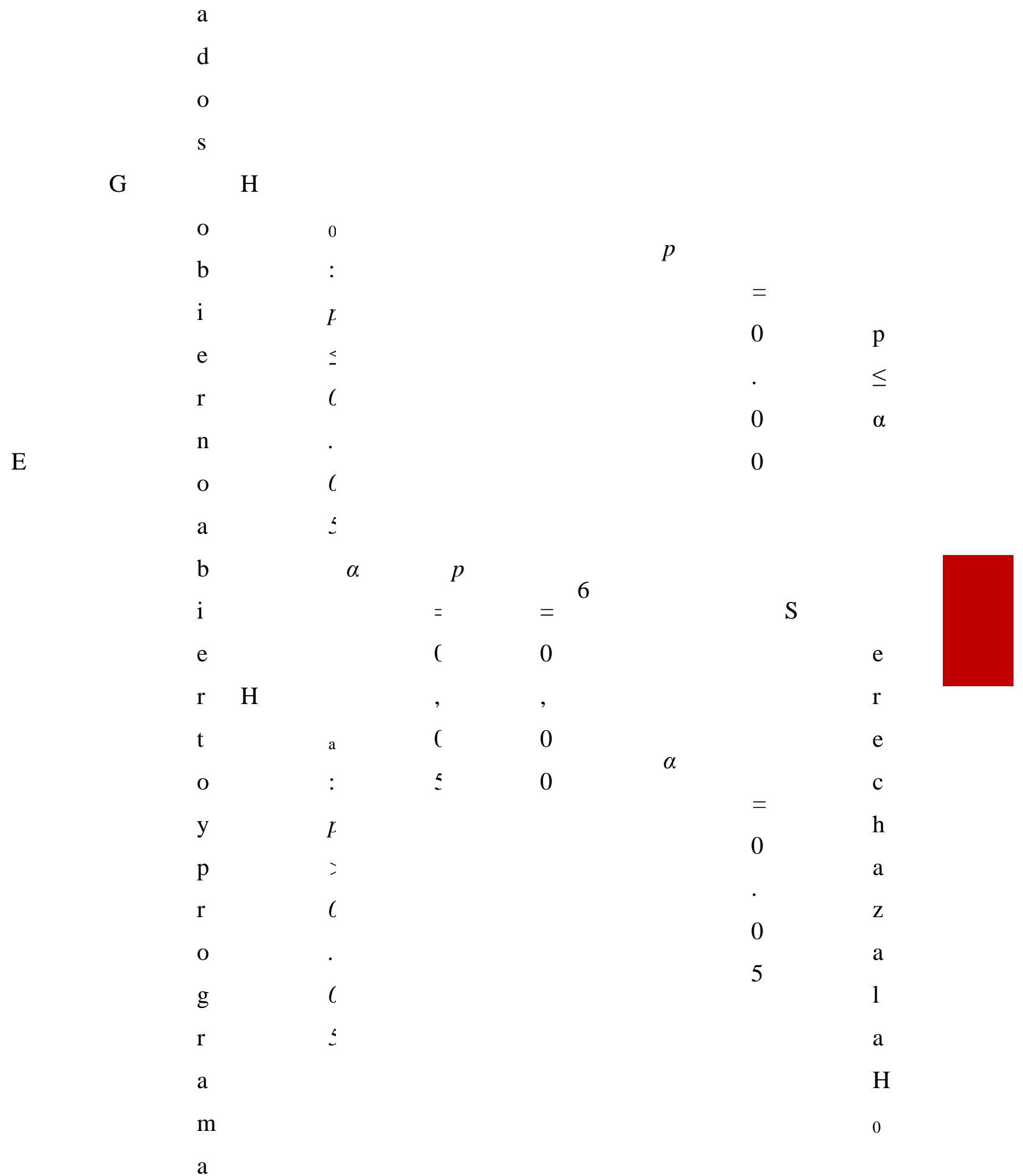


$\mathrm{u}$

d

E $\quad \mathrm{G} \quad \mathrm{H}$

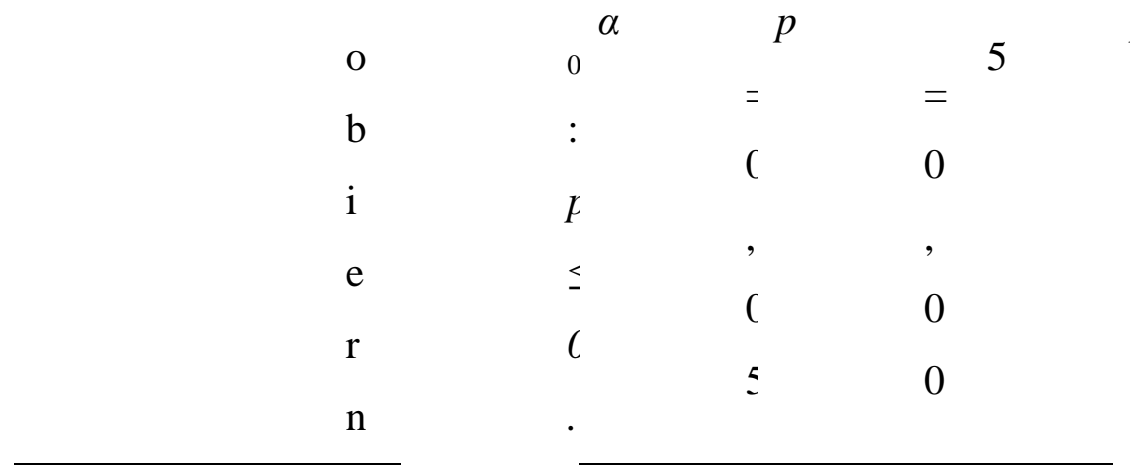
$=$

0 $\mathrm{p}$ $\leq$

0 $\alpha$ 
Fd-Rie 
De la tabla 2, se muestra el modelo predictivo $Y=f(x)$ regresión lineal simple de las seis hipótesis específicas comprobamos que, en todos los casos el valor $\mathrm{P}=0,00$ menor a la significancia $=0,05$ por lo que, se rechaza las hipótesis específicas nulas y aceptamos las hipótesis específicas de investigación. Se concluye que las sub variables de la participación de actores sociales tiene un poder explicativo significativo para explicar las dimensiones de la segunda variable gestión de programas estratégicos de salud en la región de estudio Huancavelica Perú. Los predictores encontrados fueron el Coeficiente de Correlación $\mathrm{r}^{2}$ ajustado siempre mayores al $60 \%$ (columna paso 3 ) fuertes lo que permite afirmar influencia estadística, significancia global entre las variables y dimensiones de estudio.

\section{Discusión}

Los resultados muestran una correlación alta de $84 \%$ y una erre cuadrada ajustado de $71 \%$, para determinar la influencia estadística entre variables se obtuvo con la prueba de Regresión Lineal Simple ( $\mathrm{p}=0,00$ y r=0.84) el cual señala una coherencia fuerte significativa a favor de la hipótesis planteada "la participación de actores sociales influye significativamente en la gestión de los programas estratégicos de salud en la región Huancavelica Perú”.

Dichos resultados se contrastan con la Teoría de la Acción Comunicativa de (Habermas, 2011) se concuerda respecto a los actores sociales como un conglomerado de sistemas relativamente complejos debidamente estructurados, en donde el actor como comunicador inteligente que con cada acción comunicativa coordina acciones, colabora en los procesos de la toma de decisiones a través de acuerdos argumentados que benefician a los usuarios. Se corrobora la relación directa con la Teoría Z de William Ouchi 
un modelo de gestión colaborativa integradora en el intorno de la organización, su característica la toma decisional en consenso donde se fomenta el trabajo conjunto donde prima la colaboración y el apoyo mutuo para el logro de objetivos institucionales.

(Medranda et al., 2019) afirman que es urgente motivar la participación fortaleciendo la transparencia de la información que los gobiernos están obligados a demostrar sobre su gestión, se enfatiza a través de ello se genera confianza en los entes gubernamentales para recibir colaboración. Por su parte (Idrogo et al., 2020) destacan que las autoridades deben difundir la participación masiva de la ciudad que es fundamental para mejorar los procesos y generar cambios positivos. (Rivera, 2021) resalta la importancia de la participación de la ciudadanía en apoyo a la gestión.

Así mismo (Castro et al., 2020) consideran apropiado involucrar a los ciudadanos para participar en los quehaceres organizacionales como un aliado estratégico en el proceso de planificación sobre eficiencia de los servicios públicos. (Lara, 2021) menciona a los espacios de concertación y participación, los procesos de interacción Estado y ciudadanía se han incrementado y afectan las decisiones públicas en materia de salud. En el escenario internacional (Contreras, 2018) sobre políticas de superación de la pobreza en Chile considera la medición de indicadores de gestión prioritarios para los servicios sociales tangibles en una búsqueda directa de atención de los usuarios beneficiarios.

Señala (Beltrán et al., 2020) en su estudio sobre incidencia de la gestión de presupuesto en los programas presupuestales encontraron una correlación negativa inversa de $62.4 \%$, mientras la ejecución de presupuesto se incremente, la desnutrición crónica disminuirá; según el reporte (Instituto Nacional de Estadística e Informática \& Ministerio de Economía y Finanzas, 2021) en la región Huancavelica los indicadores trazadores para la gestión de 
la salud los programas estratégicos: desnutrición crónica infantil al año 2020 fue de 31, 5 la más alta del Perú cuando el promedio nacional es de solo 12,1 sin embargo se ha logrado reducir desde el 2015 al año 2020 en solo 2,5 puntos, habiendo obtenido el último año un incremento en un punto debido a la coyuntura COVID-19 en el que las acciones se orientaron casi en su totalidad al problema y se dejaron de lado los programas estratégicos; con respecto al indicador mortalidad materna en la región se mantiene una tendencia plana los últimos 5 años y al 2020 se contó con 12 muertes maternas. (Villegas-flores, 2021) en su artículo sobre el presupuesto por resultados en la gestión administrativa encontró una relación significativa, mayor presupuesto mejor gestión administrativa en la Universidad Nacional de Moquegua.

Existen opiniones discordantes sobre participación en la gestión (Valenzuela, 2019) en el Estado de México los ciudadanos que participan en asuntos públicos se mantienen con desconfianza hacia el gobierno y la administración pública. Por otro lado (Flores Mamani et al., 2020) sobre participación de la ciudadanía en el presupuesto en Puno Perú es escasa y con muchas ausencias demostrando poco compromiso y desinterés en la gestión de recursos públicos. (Paricio-Esteban et al., 2020) sobre participación de la ciudadanía en la gestión en municipios españoles acotan el poco compromiso de transparencia y participación debido a menores niveles de cumplimiento por parte de los actores en menos del 50\%. (Melgar Carrasco, 2019) en su artículo sobre el Ministerio de Salud Perú menciona que el marco normativo del presupuesto por resultados no influye sobre reconceptualización y realineamiento, recalca en cambio existe influencia significativa sobre la reforma del sector salud y los resultados todavía serán visibles a mediano y a largo plazo. (Barros-Bastidas \& Gebera, 2020) 


\section{Conclusiones}

Se llegó a la conclusión de que las participaciones conjuntas de actores sociales influyen en la gestión de los programas estratégicos de salud en el sector público en la región Huancavelica Perú, puesto que se confirmó con la prueba estadística de Regresión Lineal a las variables valor P de 0,00 menor a la significancia de 0,05, coeficiente de correlación de 0,84 lo cual presentó un ajuste fuerte y un $\mathrm{r}^{2}$ ajustado de $71 \%$ también alta, con un nivel de confianza del $95 \%$.

Algunas implicancias respecto a la participación cuanto más alto es el grado de articulación intergubernamental entre instituciones públicas mejores logros, debido a los aliados estratégicos para trabajar en objetivos comunes, convocando a la ciudadanía y con mecanismos de trasparencia para comunicar a la sociedad civil sobre los resultados de gestión en salud que mejorarían significativamente medidos periódicamente a través de los indicadores de programas estratégicos, pero con un planeamiento estratégico a mediano y largo plazo, fortaleciendo los procesos de control con monitoreo y supervisión de actividades y con el apoyo de sistemas de información gerencial para una oportuna toma decisional para mejorar los procesos de salud, con un capital humano en salud comprometido y motivado con incentivos y bonos por su contribución a los resultados obtenidos.

Es importante incidir a los decisores en gestión en el sistema de salud Gobierno Regional, Dirección Regional de Salud, gobiernos locales implementar las políticas públicas y proponer estrategias conjuntas de intervención, actualizando los planes de desarrollo concertados y los planes estratégicos institucionales; convocatorias reuniones conjuntas de coordinación y evaluación para orientar el diseño organizacional a través de acuerdos y compromisos, el incorporar el capital humano a través de mecanismos de meritocracia, imperativo incorporar mayores recursos para 
operacionalizar los planes operativos con la finalidad de generar valor público al usuario.

\section{Referencias}

Alvarado, L., \& Agurto, H. (2013). Estadística para administración y economía (E. S. M. EIRL (ed.); 1ra ed.).

Alvarez, A., \& Gálvez, R. (2021). Presupuesto público comentado 2021 (I. P. S.A.C. (ed.); 1ra ed.).

Barros-Bastidas, C., \& Gebera, O. T. (2020). Training in research and its incidence in the scientific production of teachers in education of a public university of Ecuador. Publicaciones de La Facultad de Educacion y Humanidades Del Campus de Melilla, 50(2), 167-185. https://doi.org/10.30827/publicaciones.v50i2.13952

Barros Bastidas, C., \& Turpo Gebera, O. (2017). La formación en el desarrollo del docente investigador: Una revisión sistemática. Espacios, 38(45). http://www.revistaespacios.com/a17v38n45/17384511.html

Aracena Genao, B., Leyva Flores, R., \& del Rocío Soto Flores, M. (2020). ¿Contribuye la nueva gestión pública a mejorar el desempeño de la atención obstétrica en los hospitales públicos de México? Contaduria y Administracion, 65(1), 1-14. https://doi.org/10.22201/FCA.24488410E.2019.2109

Beltrán, K., Tarazona, J., \& Huamán, L. (2020). Incidencia de la gestión del presupuesto por resultados en los programas presupuestales sociales de los gobiernos locales de la provincia de Carhuaz-región Ancash, 2010-2015. Saber Discursivo, 1(1), 12.

Castro, D. G., De Elizagarate Gutierrez, V., Kazak, J., Szewranski, S., Kaczmarek, I., \& Wang, T. (2020). Nuevos desafíos para el perfeccionamiento de los procesos de participación ciudadana en la gestión urbana. Retos para la innovación social. Cuadernos de Gestion, 20(1), 41-64. https://doi.org/DOI: 10.5295/cdg.170751dg

Cedillo, S. P., \& Rivadeneira, E. M. (2020). Participación ciudadana en la gestión de las políticas educativas. Revista Conrado, 16, 7. 
Chura, A. (2021). Gestión por resultados en la gestión financiera. 10(051), 16.

Contreras, J. (2018). Gestión por resultados en las políticas para la superación de la pobreza en Chile. Enfoques, 16(28), 13-39. https://www.researchgate.net/profile/Jaime_Contreras4/publication/ 328461453_Gestion_por_resultados_en_las_politicas_para_la_sup eracion_de_la_pobreza_en_Chile/links/5bcf23b892851c1816bb10a f/Gestion-por-resultados-en-las-politicas-para-la-superacion-de-la-p

Dueñas, L. R., \& García, E. J. (2012). El estudio de la cultura de participación, aproximación a La demarcación del concepto. Razón y Palabra, 16(1_80), 119-135.

El Peruano, D. O. (2013). Decreto Supremo No 004-2013-PCM. El Peruano, 21.

Flores Mamani, A., Barra Quispe, D. E., \& Barra Quispe, T. L. (2020). Percepción sobre la participación ciudadana en la gestión de las políticas públicas caso Salcedo-Puno. Asociación Centro de Investigaciones y Escuela de Pensamiento Crítico Aymara “ACIEPCA, ” 2(2), 31-51.

Folleco, J. M., \& Legarda, M. A. (2020). La administración pública: Descenso de la burocracia, la nueva gestión pública y gobernanza en América Latina. International Journal of Latest Research in Humanities and Social Science (IJLRHSS), 03(10), 91-106.

Guerra, E., \& Palomino, G. del P. (2020). Modelo de gestión por resultados para mejorar la calidad de gasto en la Unidad de Gestión Educativa Local , San Martín. Ciencia Latina, 2215, 738-758.

Habermas, J. (2011). Teoría de la acción comunicativa (Editorial Cátedra (ed.); 1ra.). 
Heredia, N. L., \& Fonseca, F. (2020). Administracion colaborativa: el nuevo desafio para el administrador de empresas, como lider en las organizaciones. Journal Of Business and Entrepreurial Studies, 4, 13.

Hernández-Sampieri, R., \& Mendoza, C. (2018). Metodología de la investigación. Las rutas cuantitativa, cualitativa y mixta (S. A. de C. V. McGraw Hil Interamericana Editores (ed.); 1ra ed.).

Huanca, M. D. (2019). Gestión del presupuesto por resultados y su influencia en la calidad del gasto público en la municipalidad distrital de Chaglla - Pachitea. Gaceta Científica, 5(1), 36-38.

Idrogo, S. L., Colunche, F. L., \& Díaz, E. S. (2020). Grado de conocimientoy participación de los actores sociales sobre el presupuesto participativo del distrito de Chota.Revista Nor@ndina, 3(1), 30-37. https://doi.org/10.37518/2663-6360x2020v3n1p30

Instituto Nacional de Estadística e Informática, \& Ministerio de Economía y Finanzas. (2021). Perú indicadores de resultados de los progrmas presupuestales, 2015-2020. In Instituto Nacional de Estadística e Informática Perú.

Jara, J. L. (2018). Recursos humanos en el sector público (Pacífico Editores SAC (ed.); 1ra ed.).

Lara, M. (2021). Participación ciudadana como política de Salud pública: Una perspectiva desde los Actores Sociales de la Parroquia de Tumbaco , durante los años 2015 - 2016. Salud y Ciencias Médicas, 1,20 .

López, F. (2018). Gestion del talento humano basado en competencias en areas sociales. $\quad$ Centro Sur, $2, \quad 8$. http://centrosureditorial.com/index.php/revista/article/view/8/7 
Medranda, J. N., Torres, A., Romero, A. I., \& Caluguillin, A. P. (2019). Comunicación, ciudadanía y transparencia: Acceso a la información pública como herramienta de participación ciudadana en la gestión administrativa. Revista Ibérica de Sistemas y Tecnologías de Información, O(E26), 362-375.

Melgar Carrasco, L. M. (2019). El marco normativo de la gestión por resultados y la reforma de salud del Ministerio de Salud. LEX, 17(24), 255. https://doi.org/10.21503/lex.v17i24.1819

Milanesi, A. (2018). La gestión por resultados: una revisión de la literatura y sus desafíos en américa latina. Administración y Desarrollo, 48(1), 170-199. https://doi.org/10.22431/25005227.425

Ministerio de Salud Perú. (2016a). Programa Articulado Nutricional (p. 7428).

Ministerio de Salud Perú. (2016b). Programa Salud Materno Neonatal (p. 7428).

Montes de Oca, L. B. (2019). ¿Una nueva forma de gobernar? Modelo heurístico para analizar la participación ciudadana en la gestión pública. Revista de Gestión Pública, VIII(9), 36.

Núñez, C. R., García, L., \& Jara, D. E. (2021). La participación en la gestión cooperativa. Una experiencia cubana. Estudios Del Desarrollo Social, 9(1), 23.

Palomino, M. R. (2021). Impacto de la Gestión por Resultados en la calidad de servicio de la administración educativa. Centro Sur, E4, 16.

Paricio-Esteban, P., Bruno-Carlos, T., Alonso-Romeero, E., \& GarcíaAlcober, M. P. (2020). Webs y portales de transparencia para la participación ciudadana en la gestión de las relaciones públicas con los stakeholders locales. El Profesional de La Información, 29(3), 117. https://doi.org/10.3145/epi.2020.may.35 
Príncipe, O. A. (2021). Participación ciudadana y presupuesto participativo como una alternativa para una gobernanza efectiva para gobiernos locales en el Perú (p. 191).

Rivera, E. M. (2021). La incidencia de la participación ciudadana en el control de la gestión administrativa en el GAD municipal del cantón Ambato [Universidad Técnica de Ambato Ecuador]. In Universidad Técnica de

Ambato. http://repo.uta.edu.ec/bitstream/handle/123456789/5301/Mg.DCEv. Ed.1859.pdf?sequence $=3$

Roa, J. (2013). Gestión del deseo en la intervención social: claves estéticas y el riesgo de la participación. Revista de Trabajo Social, 83, 69-79. https://doi.org/10.7764/rts.83.69-79

Robbins, S., \& Judge, T. A. (2017). Comportamiento organizacional (Editorial: Pearson Educación de México (ed.); $17^{\mathrm{a}}$ ed.).

Valenzuela, R. E. (2019). Participación ciudadana en la gestión pública lecciones y desafios. Rabit: Jurnal Teknologi Dan Sistem Informasi Univrab, 1(1), 2019. http://www.ghbook.ir/index.php?name=فرهن هاى رسانه نوين\&option=com_dbook\&task=readonline\&book_id=13650\&page =73\&chkhashk=ED9C9491B4\&Itemid=218\&lang=fa\&tmpl=comp onent\%0Ahttp://www.albayan.ae\%0Ahttps://scholar.google.co.id/s cholar?hl=en\&q=APLIKASI+PENGENA

Vargas Merino, J. A., \& Zavaleta Chávez, W. E. (2020). La gestión del presupuesto por resultados y la calidad del gasto en gobiernos locales. Visión de Futuro, 24, No 2 (Julio-Diciembre), 37-59. https://doi.org/https://doi.org/10.36995/j.visiondefuturo.2020.24.02 .002.es 
Villegas-flores, A. D. (2021). Presupuesto por resultados y la gestión administrativa en la unidad ejecutora $\mathrm{N}^{\circ}$ 001230: Universidad Nacional de Moquegua, Moquegua, 2019. Polo de Conocimiento, 6(2), 1390-1403. https://doi.org/10.23857/pc.v6i2.2289

Zegarra, W., Olano, D., Lluncor, M., \& Cacho, A. (2021). El valor público, característica fundamental en la nueva gestión pública. Revista Científica Pakamuros, 21-30. https://doi.org/10.37787/pakamuros-unj.v9i1.160 